\title{
The runaway black hole GRO J1655-40^
}

\author{
I. F. Mirabel ${ }^{1,2}$, R. Mignani ${ }^{3}$, I. Rodrigues ${ }^{1}$, \\ J. A. Combi ${ }^{4}$, L. F. Rodríguez ${ }^{5}$, and F. Guglielmetti ${ }^{6,7}$ \\ ${ }^{1}$ Service d'Astrophysique / CEA-Saclay, 91191 Gif-sur-Yvette, France \\ 2 Instituto de Astronoma y Fsica del Espacio/Conicet, Argentina \\ 3 European Southern Observatory, Karl-Schwarzschils-Strasse 2, Garching bei München, 85740, Germany \\ 4 Instituto Argentino de Radioastronoma, C.C.5, (1894) Villa Elisa, Buenos Aires, Argentina \\ 5 Instituto de Astronoma, UNAM, Apartado Postal 3-72, 58089 Morelia, Michoacn, México \\ ${ }^{6}$ Max Planck Institute für Extraterrestrische Physik, Giessenbachstrasse, Postfach 1312, 85748, Garching, Germany \\ 7 Max Planck Institute für Plasmaphysik, Boltzmannstrasse 2, 85748, Garching, Germany
}

Received 15 July 2002 / Accepted 23 September 2002

\begin{abstract}
We have used the Hubble Space Telescope to measure the motion in the sky and compute the galactocentric orbit of the black hole X-ray binary GRO J1655-40. The system moves with a runaway space velocity of $112 \pm 18 \mathrm{~km} \mathrm{~s}^{-1}$ in a highly eccentric ( $e=0.34 \pm 0.05$ ) orbit. The black hole was formed in the disk at a distance greater than $3 \mathrm{kpc}$ from the Galactic centre and must have been shot to such an eccentric orbit by the explosion of the progenitor star. The runaway linear momentum and kinetic energy of this black hole binary are comparable to those of solitary neutron stars and millisecond pulsars. GRO J1655-40 is the first black hole for which there is evidence for a runaway motion imparted by a natal kick in a supernova explosion.
\end{abstract}

Key words. stars: individual: GRO J1655-40 - black hole physics - X-rays: binaries - astrometry

\section{Introduction}

Neutron stars are known to have large transverse motions on the plane of the sky which are believed to result from natal kicks imparted by supernova explosions. Energetic explosions have also been invoked in models of the core collapse of massive stars onto black holes. However, there have been few observations that constrain models of the physical processes by which stellar-mass black holes are formed. The measurement of a large radial velocity for the centre of mass of the black hole X-ray binary GRO J1655-40 (Orosz \& Bailyn 1997; Shahbaz et al. 1999), together with chemical elements found by Israelian et al. (1999) on the surface of the donor star, provided observational support to the idea that black holes - as neutron stars- may form in supernova explosions that impart strong natal kicks to the collapsed objects.

If a black hole is accompanied by a mass-donor star, it is possible to determine the radial velocity, proper motion, and distance of the system, from which one can derive the space velocity, track the path to the site of birth, and

Send offprint requests to: I. F. Mirabel,

e-mail: fmirabel@cea.fr

* Based on observations with the NASA/ESA Hubble Space Telescope, obtained at the Space Telescope Science Institute, which is operated by AURA, Inc. under contract No NAS 5-26555. constrain the strength of the natal kick. Presently, the most accurate proper motions of X-ray binaries are obtained following at radio wavelengths with Very Long Baseline Interferometry (VLBI) the motion in the sky of the associated compact microquasar jets, as done recently for the halo black hole binary XTE J1118+480 (Mirabel et al. 2001). This has not been possible for GRO J1655-40 because there is no VLBI calibrator nearby, and in recent years the radio counterpart faded away below the detection limit. In this context, we carried out relative astrometry of the secondary star using optical images obtained with the Hubble Space Telescope 6.3 years apart. Here we report the proper motion of GRO J1655-40 which, together with the radial velocity, allows us - taking into account the uncertainties in the distance - to determine the parameters of its runaway kinematics and galactocentric orbit.

\section{The proper motion of GRO J1655-40}

On April 1996 the field of GRO J1655-40 was observed for the first time with the WFPC2 on the Hubble Space Telescope (Tavani et al. 1996). In quiescence, the secondary star has an apparent magnitude of $m_{V} \sim 17.2$. The target had been placed at the center of the Planetary Camera (PC) chip, with a pixel size of 0.045 . Observations were obtained through the $675 \mathrm{~W}$ filter $(\lambda=6717 \AA ; \Delta \lambda=736 \AA)$. A total of 16 exposures of $40 \mathrm{~s}$ 
each were acquired on April 26th and the same sequence was repeated on April 27th but with a different telescope roll angle. Both sets of exposures were taken in groups of 4 and each group was dithered by a few pixels in both Right Ascension and Declination with respect to the others.

On June 20th 2001 a total of 18 exposures of $40 \mathrm{~s}$ each were acquired by us, with the same observational setup as in April 1996, but without any dithering between single exposures. After a pre-reduction with the standard HST pipeline reduction (debiasing, dark removal, flatfielding), groups of well-aligned exposures (i.e. with relative shift smaller than 0.01 pixel) were combined with a median filter, stacked and cosmic ray hits filtered out.

We finally ended up with 4 images for each of the two April 1996 observations and one image for the June 2001 one. All the 1996 images were then registered on the 2001 one, previously aligned in Right Ascension and Declination, by fitting the coordinate transformation between grids of reference objects. The correction for the WFPC2 geometrical distortions, optimized for the filter used (Trauger et al. 1995) was taken into account. The whole procedure was iterated until the transformation residuals were all below $\simeq 1.5 \sigma$. We finally computed the relative displacements of our target for each of the 8 independent pairs of 1996/2001 images and we averaged the results. The computed proper motion is $\mu_{\alpha}=-3.3 \pm 0.5{\text { mas } \mathrm{yr}^{-1}}^{-1}$ and $\mu_{\delta}=-4.0 \pm 0.4$ mas $\mathrm{yr}^{-1}$, corresponding to an overall

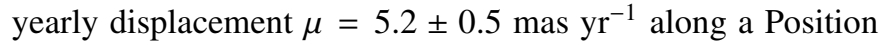
Angle of $220^{\circ} \pm 5^{\circ}$. At a distance $D(\mathrm{kpc})$ this proper motion corresponds to a transverse velocity on the plane of the sky of $(25 \pm 3) D(\mathrm{kpc}) \mathrm{km} \mathrm{s}^{-1}$. In Fig. 1 we show the path of the black hole binary on a $R$-band image of the Digitized Palomar Observatory Sky Survey II (POSS II). NGC 6242 is a well-studied open cluster at a distance of $1020 \pm 100 \mathrm{pc}$ from the Sun (Glushkova et al. 1997). Figure 1 shows that GRO J1655-40 is close to the boundary of a dark cloud (cataloged as DC344.9+2.6 in Hartley et al. 1986), which could explain the relatively large reddening of the secondary star in GRO J1655-40.

\section{The distance of GRO J1655-40}

In order to use our proper motion measurement to constrain the space velocity of the black hole, we need to know its distance. Unfortunately, as for most X-ray binaries, the distance to GRO J1655-40 is rather uncertain. In the following we discuss the observational constrains to the distance of this source.

\subsection{The relativistic distance}

It is widely believed that the distance of GRO J1655-40 was well determined solely from the kinematics of the two-sided radio jets. This is not true. The relativistic time delay of the motion of the ejecta in the sky is given by the two equations (Mirabel \& Rodríguez 1994):

$\mu_{\mathrm{r}, \mathrm{a}}=\frac{\beta \sin (\theta)}{1 \pm \beta \cos (\theta)} \frac{c}{D}$ where $\mu_{\mathrm{r}}$ and $\mu_{\mathrm{a}}$ are the proper motions of the receding and approaching jets. In this system of two equations there are three unknowns: the angle with the line of sight of the jet axis $\theta$, the velocity of the jets $\beta=\frac{v}{c}$, and the distance $D$. We point out that using solely the observations at radio wavelengths by Hjellming \& Rupen (1995) it is not possible to solve these equations, unless one assumes a value for one of the three variables $\theta, \beta$, or D. Hjellming \& Rupen (1995) assumed $\theta=85^{\circ} \pm 2^{\circ}$, from which one derives $\beta=\frac{v}{c}=0.92$ and $D=$ $3.2 \mathrm{kpc}$. As already noticed by Orosz \& Bailyn (1997), in this case the axis of the jet and the axis of the orbital plane differ by $\sim 15^{\circ} \pm 2^{\circ}$.

We point out that the assumption that the jet axis is parallel to the axis of the orbital plane is equally consistent with the observations at radio wavelengths. The jet axis and the orbital plane must be coupled, since the period of rotation of the jets about the jet axis (Hjellming \& Rupen 1995) is - within the uncertainties -, the same as the 2.6 day orbital period of the binary (Orosz \& Bailyn 1997). If one assumes that the twin jets are perpendicular to the orbital plane of the binary, from $\mu_{\mathrm{r}}=$ 45 mas/day and $\mu_{\mathrm{a}}=54$ mas/day (Hjellming \& Rupen 1995), $\theta=70.2^{\circ} \pm 1.9^{\circ}$ (Greene et al. 2001) result $\beta=\frac{v}{c}=0.27 \pm 0.03$ (where $v$ is the velocity of the jets and $c$ the speed of light) and a distance $D=893 \pm 100 \mathrm{pc}$. Under this assumption, the distance would be a factor 3.5 closer than commonly assumed, the jet velocity would be similar to that in SS433, and GRO J1655-40 would not be a superluminal source.

In summary, from the data at radio wavelengths and the system of two Eq. (1) with three unknowns one can only derive with certainty a relativistic upper limit (Mirabel \& Rodríguez 1999) given by $D \leq c /\left(\mu_{\mathrm{r}} \mu_{\mathrm{a}}\right)^{-1 / 2}=3.5 \mathrm{kpc}$.

\subsection{The distance and the interstellar matter along the line of sight}

A distance for GRO J1655-40 was proposed on the basis of optical (Bailyn et al. 1995) and X-ray (Greiner et al. 1995; Ueda et al. 1998a,b) measurements of the column of interstellar matter in the line of sight, under the assumption that the absorbing material is distributed homogeneously between the source and the observer. However, GRO J1655-40 is at relatively high Galactic latitude (Galactic longitude and latitude $l=345.0^{\circ}$, $\left.b=+2.2^{\circ}\right)$ in the Scorpius region of the sky which contains rather clumpy optical dark clouds in the foreground (see Fig. 1), that have $60 \mu \mathrm{m}$ and $100 \mu \mathrm{m}$ IRAS counterparts of dust emission. From a study of the reddening undergone by the stars that are at distances between $700 \mathrm{pc}$ and $1900 \mathrm{pc}$, it is known that most of the reddening in this region of the sky occurs in the local arm within 700 pc from the Sun (Crawford et al. 1989).

On the other hand, a kinematic distance was proposed from the radial velocity of absorption features in the $\mathrm{HI} \lambda 21 \mathrm{~cm}$ line spectrum (Tingay et al. 1995). However, it is known that in this region of the sky at distances $\leq 1900 \mathrm{pc}$ there are clouds with anomalous velocities of up to $-50 \mathrm{~km} \mathrm{~s}^{-1}$ (Crawford et al. 1989). Therefore, it is not possible to derive the distance of GRO J1655-40 only from the column density and/or kinematics of the interstellar matter in the line of sight. 


\subsection{Constrains on the distance from the properties of the secondary star}

The main argument in favor of the canonical distance of $\sim 3.2 \mathrm{kpc}$ is based on the flux, color and size of the secondary. The radius is inferred from the photometric light curves which provide evidence that the secondary fills its Roche lobe (Orosz \& Bailyn 1997; Shahbaz et al. 1999; Soria et al. 2000; van der Hooft et al. 1997, 1998; Phillips et al. 1999). From the optical spectrum (Orosz \& Bailyn 1997) and interstellar absorption (Horne et al. 1996) a temperature is derived, which together with the radius provides an intrinsic luminosity. In the most recent model by Beer \& Podsiadlowski (2002) the secondary would have a luminosity of $21 \pm 6.0 \mathrm{~L}_{\odot}$, which is consistent with a distance $\geq 2 \mathrm{kpc}$. These authors estimate masses for the black hole $M_{\mathrm{BH}}=5.4 \pm 0.3 M_{\odot}$ and for the donor star $M_{*}=1.45 \pm 0.35 M_{\odot}$.

We point out that the secondary star in quiescence has an apparent magnitude $m_{V}=17.12$, it has been classified as an F3 IV-F6 IV sub-giant (Orosz \& Bailyn 1997), and along the line of sight there is an interstellar absorption $A_{V}=3.1 \times$ $E(B-V)=4.03 \mathrm{mag}$ (Horne et al. 1996). A sub-giant star of this spectral type has a mean intrinsic magnitude $M_{V} \sim 3.2 \pm$ 0.2 mag (Popper 1980; Aller et al. 1982), and it would be at a distance $D=950 \pm 150 \mathrm{pc}$. Alternatively, if the secondary were a main sequence star of spectral type F5 V star (Regős et al. 1998), for an absorption $A_{V}$ in the range of 3.3-4.3 mag the distance would be in the range of $800-1250 \mathrm{pc}$. However, it may be incorrect to attribute the absolute magnitudes of isolated stars to secondary stars in X-ray binaries with the same spectral type.

It was pointed out by Beer \& Podsiadlowski (private communication) that the lower luminosity and temperature implied by a distance of $\sim 1 \mathrm{kpc}$ would require a higher mass ratio with much reduced masses for the compact object $\left(\sim 3.2 M_{\odot}\right)$ and secondary star $\left(\sim 0.1 M_{\odot}\right)$. But the absorption spectrum of the secondary seems to rule out an M type star of such low mass or a K-type star with mass $0.6 \leq M_{\odot}$. The spectra of stars with $T_{\text {eff }} \leq 5000 \mathrm{~K}$ have different signatures, such as strong molecular bands. This was the case in GRS 1915+105 where $K$ band spectroscopy (Greiner et al. 2001) revealed CO molecular bands rendering invalid the classification of the donor as a main sequence star.

We point out that there have been analogous uncertainties about the nature of the secondary in the X-ray binary LMX-3; the proposition that it is a main sequence star (Cowley et al. 1983; van der Klis et al. 1985) has recently been challenged by Soria et al. (2001) who argue that it is a sub-giant. Furthermore in GRO J1655-40, the contribution to the optical flux from: 1) the accretion disk detected in the X-rays at times when it was believed that the source was in optical quiescence (Garcia et al. 2001), and 2) possible non-thermal processes (e.g. synchrotron jets) that may be associated with the polarization observed in the optical flux (Gliozzi et al. 1998) is not known. In this context, for the scope of the present study we leave as an open question the issue of the distance of GRO J1655-40.
Table 1. Parameters of the runaway motion and galactocentric orbit of GRO J1655-40, for two extreme values of the distance. $V_{\text {run }}$ is the runaway velocity in three dimensions after correction for differential Galactic rotation. $e$ is the eccentricity of the galactocentric orbit, $Z_{\max }$ is the maximal height above the Galactic plane, and $r_{\max }$ and $r_{\min }$ are the maximal and minimal galactocentric distances. The runaway linear momentum $p$ and kinetic energy $T_{\text {kin }}$ of the binary system are computed according to the masses given in the text.

\begin{tabular}{ccc}
\hline \hline$D[\mathrm{kpc}]$ & 0.9 & 3.2 \\
\hline$V_{\text {run }}\left[\mathrm{km} \mathrm{s}^{-1}\right]$ & 130 & 93 \\
\hline$e$ & 0.39 & 0.29 \\
\hline$z_{\max }[\mathrm{kpc}]$ & 0.05 & 0.15 \\
\hline$r_{\max }[\mathrm{kpc}]$ & 13.8 & 7.2 \\
\hline$r_{\min }[\mathrm{kpc}]$ & 6.0 & 3.9 \\
\hline$p\left[M_{\odot} \mathrm{km} \mathrm{s}^{-1}\right]$ & 430 & 637 \\
\hline$T_{\text {kin }}[\mathrm{erg}]$ & $5.6 \times 10^{47}$ & $5.9 \times 10^{47}$ \\
\hline
\end{tabular}

\section{Space velocity and galactocentric orbit}

As shown below, the parameters of the runaway motion and galactocentric orbit of GRO J1655-40 are essentially the same for distances in the range of $0.9-3.2 \mathrm{kpc}$. The tangential velocity is

$V_{\mathrm{t}}=(25 \pm 3) D_{\mathrm{kpc}} \mathrm{km} \mathrm{s}^{-1}$,

where $D_{\mathrm{kpc}}$ is the distance in $\mathrm{kpc}$. The radial velocity with respect to the Sun is $-142.4 \pm 1.5 \mathrm{~km} \mathrm{~s}^{-1}$ (Orosz \& Bailyn 1997; Shahbaz et al. 1999).

Using values of the position, distance, proper motion and radial velocity, the Galactic orbit of GRO J1655-40 can be computed using a Galactic gravitational potential model (Johnston et al. 1995). The velocity components $U, V$, and $W$ directed to the Galactic centre, rotation direction, and north Galactic pole are derived using Johnson \& Soderblom (1987)'s equations of transformation, and assuming the sun moves $\left(U_{\odot}, V_{\odot}, W_{\odot}\right)=$ $(9,12,7) \mathrm{km} \mathrm{s}^{-1}$ relative to the local standard of rest (lsr) (Mihalas \& Binney 1981). Two different orbits were computed for two extreme values of the distance: for $D=0.9 \mathrm{kpc}$ we obtain $(U, V, W)=(-133 \pm 2,27 \pm 2,1 \pm 3)$ and for $D=3.2 \mathrm{kpc}$, $(U, V, W)=(-121 \pm 18,-33 \pm 8,3 \pm 8)$. These two sets of values are rather different from the mean values that characterize the kinematics of stars that belong to the halo, and the thin and thick disk of the Galaxy (Chiba \& Beers 2000). The runaway velocities $V_{\text {run }}$ were computed for the two possible extreme distances of $0.9 \mathrm{kpc}$ and $3.2 \mathrm{kpc}$ (see Table 1), after subtracting the Galactic differential rotation given by the model for the corresponding position of the source in the Galactic disk.

The runaway linear momentum $p$ and kinetic energy $T_{\text {kin }}$ were computed assuming $M_{\mathrm{BH}}=5.4 \pm 0.3 M_{\odot}$ and $M_{*}=1.45 \pm$ $0.3 M_{\odot}$ for a distance $D=3.2 \mathrm{kpc}$ (Beer \& Podsiadlowski 2002), and $M_{\mathrm{BH}}=3.2 M_{\odot}$ and $M_{*}=0.1 M_{\odot}$ for a distance $D=0.9 \mathrm{kpc}$ (Beer \& Podsiadlowski, private communication).

The parameters of the runaway motion and galactocentric orbit of GRO J1655-40 are given in Table 1. In Fig. 2 are represented the Galactocentric orbits. For a given Galactic potential the orbital parameters do not change as a function of 


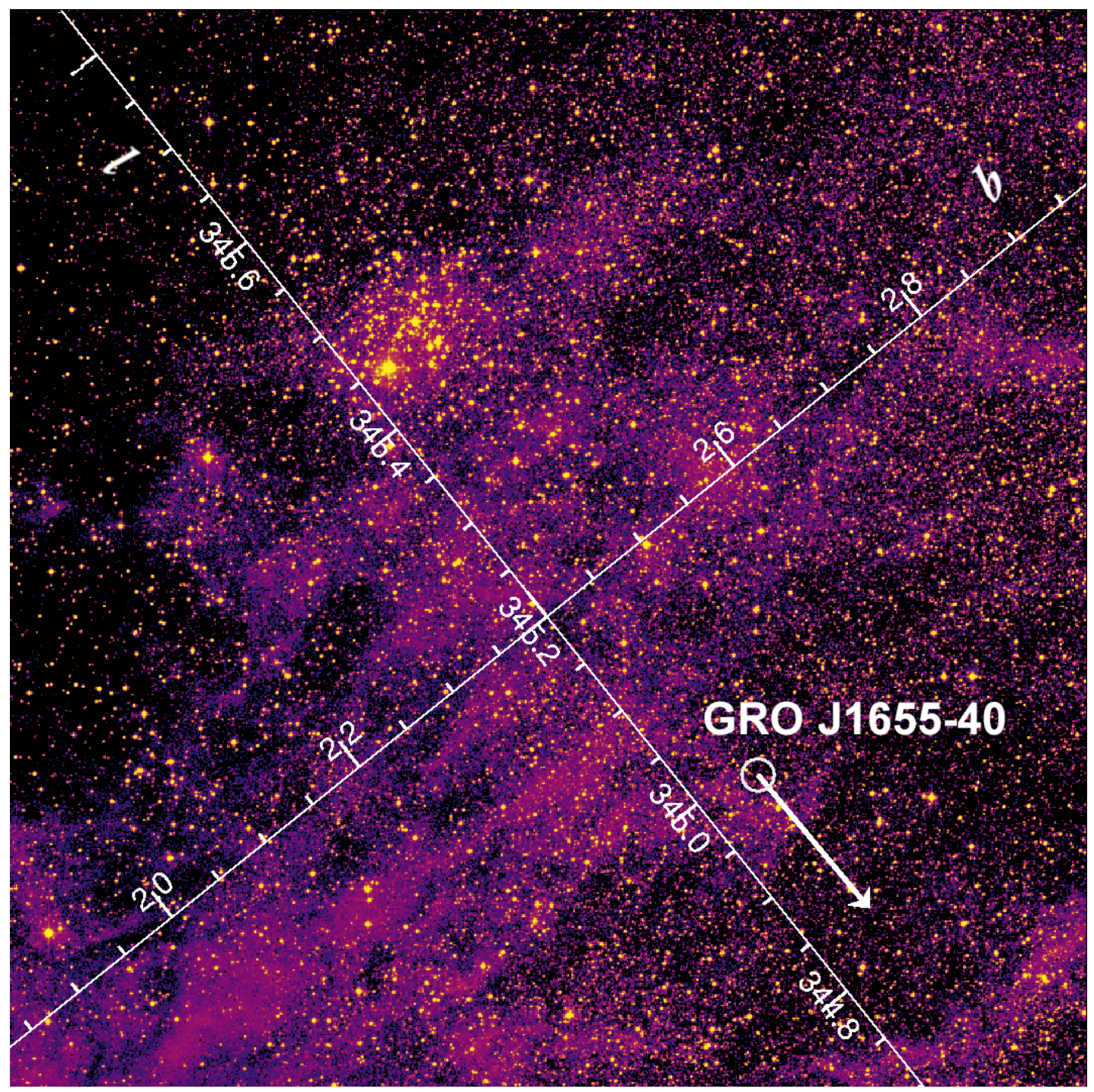

Fig. 1. Position of GRO J1655-40 on a $R$ band image from the Digitized Palomar Observatory Sky Survey II (POSS II), in Galactic coordinates. The arrow shows the direction of the motion at a rate of $5.2 \pm 0.5$ mas yr$^{-1}$ measured with the Hubble Space Telescope. Most of the stars around $l=345.44^{\circ}, b=+2.43^{\circ}$ belong to the open cluster NGC 6242 which is at a distance of $1 \pm 0.1 \mathrm{kpc}$ from the sun. Because of the uncertainty in the distance to GRO J1655-40 the association with the cluster cannot be assessed.
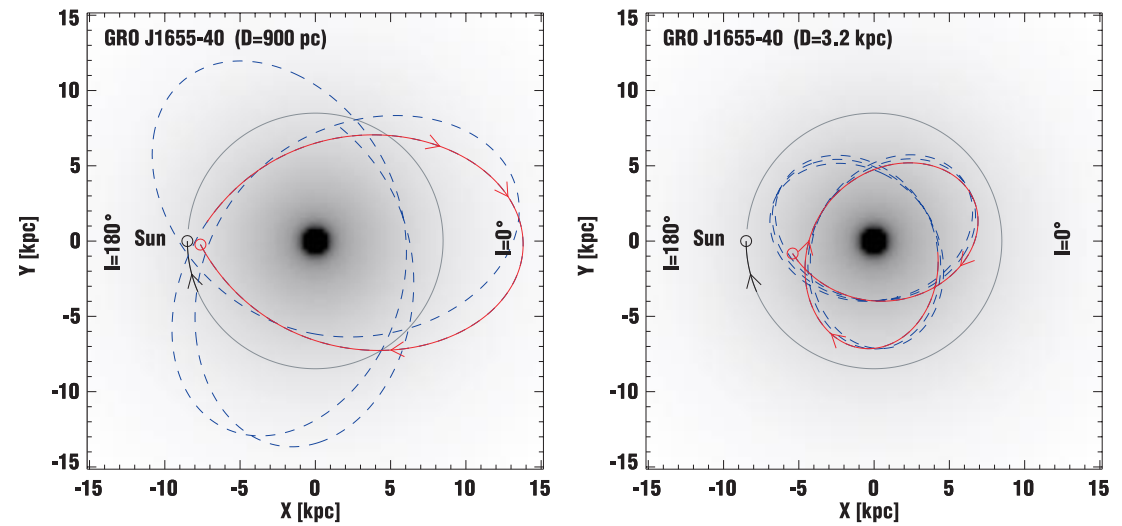

Fig. 2. Galactocentric orbits of GRO J1655-40 viewed from above the Galactic plane. The orbital plane is almost parallel to the Galactic disk and the source never reaches heights greater than $150 \mathrm{pc}$. On the left is shown the orbit obtained for an heliocentric distance $D=0.9 \mathrm{kpc}$, and on the right, for $D=3.2 \mathrm{kpc}$. GRO J1655-40 never penetrates the Galactic bulge. Plotted in dashed blue line is 1 Gyr of backwards integration, and in red the past 230 Myrs. 
time. The selection of different Galactic potentials from the current models introduce a scatter in the values of the orbital parameters smaller than $10 \%$. In fact, the errors in the parameters listed in Table 1 are largely dominated by the uncertainty in the distance. For the range of distances $0.9-3.2 \mathrm{kpc}$, the galactocentric orbit is highly eccentric $(e=0.29-0.39)$, the source always moves within a maximum height of $150 \mathrm{pc}$ from the Galactic plane, and since the minimum perigalactic distance is $3.9 \mathrm{kpc}$ it never reaches the Galactic bulge.

Clearly, GRO J1655-40 does not move in a halo orbit as XTE J1118+480 (Mirabel et al. 2001). GRO J1655-40 must have been born in the Galactic plane at a galactocentric distance $\geq 3.8 \mathrm{kpc}$. The runaway linear momentum and kinetic energy of the binary system are comparable to those of solitary neutron stars and millisecond pulsars (Toscano et al. 1999).

\section{Conclusion}

From the proper motion and radial velocity of GRO J1655-40 irrespective of the uncertainties in the distance - we conclude the following:

1. The X-ray binary has a runaway velocity of $112 \pm$ $18 \mathrm{~km} \mathrm{~s}^{-1}$, probably imparted by a natal explosion during the formation of the black hole.

2. The linear momentum of the binary system is $538 \pm$ $100 M_{\odot} \mathrm{km} \mathrm{s}^{-1}$, which is comparable to that found in solitary neutron stars and millisecond pulsars. This suggests that the relatively low-mass black hole $\left(\leq 6 M_{\odot}\right)$ in GRO J1655-40 may have been formed through a neutron star phase.

3. The kinetic energy of the system is $(5.8 \pm 0.4) \times 10^{47}$ ergs, namely, $\sim 6 \times 10^{-4}$, that of a typical supernova.

4. The galactocentric orbital plane is almost parallel to the Galactic disk and the Galactic pericentre $\geq 3 \mathrm{kpc}$, which indicates that the black hole in GRO J1655-40 is the remnant of a massive star born in the Galactic disk.

5. Contrary to the binary XTE J1118+480 which harbors a black hole born in the halo (Mirabel et al. 2001), in the case of GRO J1655-40 there is unambigous kinematical evidence that the black hole was born in the disk and received a natal kick, most likely from a supernova explosion (Israelian et al. 1999).

Acknowledgements. We thank J. Casares, P. Podsiadlowski, M.E. Beer, J.-P. Lasota, S. Corbel, P. Benaglia, A. Piatti, and E. Gourgoulhon for help and discussions on different aspects of this work. J.A.C. and I.F.M. are members of the Consejo Nacional de Investigaciones Científicas y Técnicas of Argentina, and I.R. is a fellow of the Conselho Nacional de Desenvolvimento Cienífico e Tecnológico of Brazil. I.F.M. acknowledges support from PIP 0049/98 and Fundación Antorchas.

\section{References}

Aller, L. H., Appenzeller, I., Baschek, B., et al. (eds.) 1982, Landolt-Börnstein: Numerical Data and Functional Relationships in Science and Technology - New Series Gruppe/Group 6
Astronomy and Astrophysics vol. 2 Schaifers/Voigt: Astronomy and Astrophysics / Astronomie und Astrophysik Stars and Star Clusters / Sterne und Sternhaufen

Bailyn, C. D., Orosz, J. A., Girard, T. M., et al. 1995, Nature, 374, 701

Beer, M. E., \& Podsiadlowski, P. 2002, MNRAS, 331, 351

Chiba, M., \& Beers, T. C. 2000, AJ, 119, 2843

Cowley, A. P., Crampton, D., Hutchings, J. B., Remillard, R., \& Penfold, J. E. 1983, ApJ, 272, 118

Crawford, I. A., Barlow, M. J., \& Blades, J. C. 1989, ApJ, 336, 212

Garcia, M. R., McClintock, J. E., Narayan, R., et al. 2001, ApJ, 553, L47

Gliozzi, M., Bodo, G., Ghisellini, G., Scaltriti, F., \& Trussoni, E. 1998, A\&A, 337, L39

Glushkova, E. V., Zabolotskikh, M. V., Rastorguev, A. S., Uglova, I. M., \& Fedorova, A. A. 1997, Astron. Lett., 23, 71

Greene, J., Bailyn, C. D., \& Orosz, J. A. 2001, ApJ, 554, 1290

Greiner, J., Cuby, J. G., McCaughrean, M. J., Castro-Tirado, A. J., \& Mennickent, R. E. 2001, A\&A, 373, L37

Greiner, J., Predehl, P., \& Pohl, M. 1995, A\&A, 297, L67

Hartley, M., Tritton, S. B., Manchester, R. N., Smith, R. M., \& Goss, W. M. 1986, A\&As, 63, 27

Hjellming, R. M., \& Rupen, M. P. 1995, Nature, 375, 464

Horne, K., Harlaftis, E. T., Baptista, R., et al. 1996, IAU Circ., 6406, 2

Israelian, G., Rebolo, R., Basri, G., Casares, J., \& Martin, E. L. 1999, Nature, 401, 142

Johnson, D. R. H., \& Soderblom, D. R. 1987, AJ, 93, 864

Johnston, K. V., Spergel, D. N., \& Hernquist, L. 1995, ApJ, 451, 598

Mihalas, D., \& Binney, J. 1981, Galactic astronomy: Structure and kinematics, 2nd ed. (San Francisco, CA, W. H. Freeman and Co.) 608

Mirabel, I. F., Dhawan, V., Mignani, R. P., Rodrigues, I., \& Guglielmetti, F. 2001, Nature, 413, 139

Mirabel, I. F., \& Rodríguez, L. F. 1994, Nature, 371, 46

Mirabel, I. F., \& Rodríguez, L. F. 1999, ARA\&A, 37, 409

Orosz, J. A., \& Bailyn, C. D. 1997, ApJ, 477, 876

Phillips, S. N., Shahbaz, T., \& Podsiadlowski, P. 1999, MNRAS, 304, 839

Popper, D. M. 1980, ARA\&A, 18, 115

Regős, E., Tout, C. A., \& Wickramasinghe, D. 1998, ApJ, 509, 362

Shahbaz, T., van der Hooft, F., Casares, J., Charles, P. A., \& van Paradijs, J. 1999, MNRAS, 306, 89

Soria, R., Wu, K., \& Hunstead, R. W. 2000, ApJ, 539, 445

Soria, R., Wu, K., Page, M. J., \& Sakelliou, I. 2001, A\&A, 365, L273

Tavani, M., Fruchter, A., Zhang, S. N., et al. 1996, ApJ, 473, L103

Tingay, S. J., Jauncey, D. L., Preston, R. A., et al. 1995, Nature, 374, 141

Toscano, M., Sandhu, J. S., Bailes, M., et al. 1999, MNRAS, 307, 925

Trauger, J. T., Vaughan, A. H., Evans, R. W., \& Moody, D. C. 1995, in Calibrating Hubble Space Telescope. Post Servicing Mission. Proceedings of a Workshop held at the Space Telescope Science Institute, in Baltimore, Maryland, May 15-17, 1995, ed. A. Koratkar, \& C. Leitherer (Space Telescope Science Institute), Baltimore, Maryland. LC \#: QB500.268 .C34 1995. ISBN \#: NONE., 379

Ueda, Y., Inoue, H., Tanaka, Y., et al. 1998a, ApJ, 492, 782

Ueda, Y., Inoue, H., Tanaka, Y., et al. 1998b, ApJ, 500, 1069

van der Hooft, F., Groot, P. J., Shahbaz, T., et al. 1997, MNRAS, 286, L43

van der Hooft, F., Heemskerk, M. H. M., Alberts, F., \& van Paradijs, J. 1998, A\&A, 329, 538

van der Klis, M., Clausen, J. V., Jensen, K., Tjemkes, S., \& van Paradijs, J. 1985, A\&A, 151, 322 